\title{
The Application and Modeling
}

\section{for Conditional Heteroscedasticity Time Series}

\author{
Wenfang Su (Corresponding author) \\ Department of Science, Yanshan University \\ 438 West of He Bei Avenue, Qinhuangdao 066004, China \\ E-mail:suwenfang0228@126.com \\ Rui Shan \& Jun Zhang \\ Department of Science, Yanshan University \\ 438 West of He Bei Avenue, Qinhuangdao 066004, China \\ Yan Gao \\ Jangheliu Senior High School \\ Hebei 053500, China
}

\begin{abstract}
This article mainly presents the fundamental theory, model and application of conditional heteroscedasticity residual sequence. And it also gives detailed, scientific and exact analysis and research on a financial security example. Then summarizing a conclusion: Financial Securities follows specific rules and tracks through above study. The research indicates that $\mathrm{ARCH}$ model only applies to a short-term, auto-correlative heteroscedastic function, whereas the amended GARCH model has the opposite result, that is, GARCH fits a long-term, auto-correlative heteroscedastic function. Meanwhile, SAS program presents more intuitive, exact tables and figures. All analysis and results show that AR (m)-GARCH fits well.
\end{abstract}

Keywords: Time series, Heteroscedastic function, ARCH, GARCH, SAS

\section{Introduction}

In recent years, with computing technology and signal processing technology, the theory and methods of time series analysis has been refined greatly, especially in the parameter estimation algorithm, model structure identification and intellectual computing technology integration and so on. Furthermore it gains fruitful achievement in these fields and covers an increasingly wide range of applications, and the results are at a high-level of level. For example, in the field of control engineering, motion control system for time series analysis modeling and forecast; in Internet technologies, network traffic analysis of time series model; theoretical studies in the database, data mining of time series methods; in electronic information field, random signals in time series modeling and analysis; in the field of biological engineering, DNA sequence analysis and calculation; in the field of biomedical engineering, biomechanical and electrical signals in time series analysis; in mechanical fault diagnosis study, non-destructive testing signals in time series analysis; fine chemical control in the use of time series spectral analysis techniques and so on.

Although the time series offers a variety of different models to fit the actual problem, and make short-term prediction, such as commonly used model has ARMA, ARIMA, etc... We are familiar with ARMA, ARIMA model and think its residual sequence is white noise sequence which meets $E\left(\varepsilon_{t}\right)=0$; and also meets $D \varepsilon_{t}=\gamma(0)=\delta^{2}$, where, $\delta^{2}$ is a constant. However, when Engle did research on the UK inflation rate in 1982, he surprisingly found that the classic ARIMA model has failed to achieve the desired effect of the fitting. After careful study on sequence of residuals, he discovered a problem in some time series of residuals with heteroscdasticity. In recent year scholars found many financial time series has emerged of the nature of heteroscedasticity in practice, and usually there is a positive relationship between the standard deviation and the level. That is, with low levels of sequence, the sequence of fluctuations small, with high levels of sequence, the sequence of fluctuations in large. Although we have made appropriate assumptions to heteroscedastic function, a lot of practice has proved that this assumption is too simplistic. Thus, in order to estimate heteroscedastic function more accurately, Engle made conditional heteroscedasticity ARCH and GARCH models. 


\section{ARCH model}

The full name of ARCH model is autoregressive conditional heteroscedasticity model (autoregressive conditional heteroscedastic), it is made by Robert F. Engle, who is an American statistician, economist measurement. Set $\left\{x_{t}, t=1,2, \ldots\right\}$ is a time series, called the model with the following structure for the q-order Autoregressive Conditional Heterocedasticity Model, easily recorded as $\mathrm{ARCH}(\mathrm{q})$, its complete structure as follows:

$$
\begin{aligned}
& x_{t}=f\left(t, x_{t-1}, x_{t-2}, \ldots\right)+\varepsilon_{t} \\
& \varepsilon_{t}=\sqrt{h_{t}} e_{t} \\
& h_{t}=\omega+\sum^{q} \lambda_{j} \varepsilon_{t-j}^{2}
\end{aligned}
$$

Where, $f\left(t, x_{t-1}, x_{t-2}, \ldots\right)$ is the regression ${ }^{j}$ function of Auto-Regressive Model of $\left\{x_{t}\right\} ; h_{t}=\omega+\sum^{q} \lambda_{j} \varepsilon_{t-j}^{2}$ is heteroscedasticity function; $e_{t} \stackrel{i . i . d}{\sim} N(0,1) ;\left\{\varepsilon_{t}\right\}$ is white noise heteroscedasticity residual sequence=1which has $E\left(\varepsilon_{t}\right)=0$.

\section{GARCH model}

After ARCH model was amended by Bollerslev, he proposed the GARCH model (generalized autoregressive conditional heteroscedastic), easily recorded as $\operatorname{GARCH}(p, q)$, its structure as follows:

$$
\begin{aligned}
x_{t} & =f\left(t, x_{t-1}, x_{t-2}, \ldots\right)+\varepsilon_{t} \\
\varepsilon_{t} & =\sqrt{h_{t}} e_{t} \\
h_{t} & =\omega+\sum^{p} \eta_{i} h_{t-i}+\sum^{q} \lambda_{j} \varepsilon_{t-j}^{2}
\end{aligned}
$$

Where, $f\left(t, x_{t-1}, x_{t-2}, \ldots\right)$ is the regression'=function of $=\left\{x_{t}\right\} ; e_{t} \sim{ }_{i . i . d} N(0,1) ; h_{t}=\omega+\sum^{p} \eta_{i} h_{t-i}+\sum^{q} \lambda_{j} \varepsilon_{t-j}^{2}$ is the GARCH model heteroscedasticity function, $\left\{\varepsilon_{t}\right\}$ is white noise heteroscedasticity residual sequence which has $E\left(\varepsilon_{t}\right)=0$.

\subsection{GARCH model constraints}

GARCH model in the use of modeling practical problems should pay attention: its effective use must meet the following two constraints.

Condition 1: parameters of non-negative

$$
\omega>0, \eta_{i} \geq 0, \lambda_{j} \geq 0
$$

Condition 2: parameters have limit

$3.2 \operatorname{GARCH}(p, q)$ modeling thought $\sum_{i=1}^{p} \eta_{i}+\sum_{j=1}^{q} \lambda_{j}<1$

GARCH model fitted to follow the six steps:

Step1.According to observations of the nature of sequences, fitting regression model;

Step2.Test the residual sequence autocorrelation through statistic DW;

Step3.The use of statistic PQ and LM for heteroscedasticity autocorrelation test;

Step4.Through the DW test results, residual autocorrelation sequence diagram as well as PQ and LM test statistic for the fitted-order model fitting;

Step5.The use of maximum likelihood estimation method to estimate the unknown parameters;

Step6.Finally the use of Bear-Jarque normality test statistic for testing the validity of the model.

\section{AR (m)-GARCH}

When the GARCH model of regression function $f\left(t, x_{t-1}, x_{t-2}, \ldots\right)$ can not extract the sequence $\left\{\varepsilon_{t}\right\}$ of the relevant information fully, the residual sequence may have a nature of auto-correlation, rather than pure randomness. At this point the need for $\left\{\varepsilon_{t}\right\}$ first fitting autoregressive model, and then inspecting autoregressive residual sequence $\left\{v_{t}\right\}$ whether meets $D \varepsilon_{t}=\gamma(0)=\delta^{2}$ or not. If $\left\{v_{t}\right\}$ is heteroscedasticity, GARCH model is fitted. This model called AR (m)-GARCH (p, q) model, its structure as follows:

$$
\begin{aligned}
x_{t} & =f\left(t, x_{t-1}, x_{t-2}, \ldots\right)+\varepsilon_{t} \\
\varepsilon_{t} & =\sum_{k=1}^{m} \beta_{k} \varepsilon_{t-k}+v_{t} \\
v_{t} & =\sqrt{h_{t}} e_{t}
\end{aligned}
$$




$$
h_{t}=\omega+\sum_{i=1}^{p} \eta_{i} h_{t-i}+\sum_{j=1}^{q} \lambda_{j} v_{t-j}^{2}
$$

Where, $f\left(t, x_{t-1}, x_{t-2}, \ldots\right)$ is the regression function of $\left\{x_{t}\right\} ; \underset{t}{\stackrel{i . i . d}{\sim}} N(0,1)$

\section{SAS Program Results and Discussion}

Table 1 shows security data about Reserve Bank of Australia from 1969, 1 to 1994, 9. Then we will analyze these data and fit an appropriate model to dignify them. Table 2 gives the DW test results. The results show residual sequence has obvious positive auto-correlativity. Parameter estimation demonstrates the regressive model parameters are all remarkable. From table 3, we know residual sequence autocorrelation has a long-term auto-correlativity, and the coefficient decreases slowly. Table 4 demonstrates PQ and LM test results. The results show remarkable heteroscedastic nature and long-term relation. From table 5, we can make sure that the value of different parameters in the AR (2)-GARCH $(1,1)$. Last we can acquire the formula of AR (2)-GARCH $(1,1)$ model, as follows:

$$
\begin{aligned}
& x_{t}=0.0358 t+u_{t} \\
& u_{t}=1.0754 u_{t-1}-0.081 u_{t-2}+\varepsilon_{t} \\
& \varepsilon_{t}=\sqrt{h_{t}} a_{t}, a_{t}^{i . i . d} N(0,0.35041) \\
& h_{1}=0.34+(2.554 E-23) \varepsilon_{t-1}^{2}+0.0298 h_{t-1}
\end{aligned}
$$

Figure 1 shows the curve track of $\left\{x_{t}\right\}$. From figure 1, we can see the curve presents remarkable linear increasing tendency, and the range of fluctuation increases with time extends. So we fit the linear regressive model. Figure 2 shows the curve of AR (2)-GARCH $(1,1)$. Comparing with figure 1, we see the two curves are quite similar.

\section{Conclusions}

From the above example of analysis and study, we acquire such conclusion: the AR (m)-GARCH model usually applies to Financial Sequence which has a long-term , auto-correlative heteroscedasticity function, the AR(2)-GARCH(1, 1) fits well from the two figures. So under the heteroscedasticity conditions, we find a better model to fit this kind of practical problems.

\section{References}

Gao, Huixuan. (1998). SAS System, SAS/ETS Software User Manual. Beijing: China Statistics Press. pp. 116-153. James D. Hamilton. (1999). Time Series Analysis. Beijing: China Social Science Press. pp. 799-819.

Pan, Hongyu. (2006). Time Series Analysis. Beijing: Foreign Economic and Trade University Press.

Wang, Yan. (2005). Application of Time Series Analysis [M]. Beijing: China People University Press. pp.167-207.

Wang, Zhenlong. (2007). Time Series Analysis. Beijing: Science Press.

Zhang, Xiaolong. (2007). Inventory Control under Temporal Demand Heterosedasticity. European Journal of Operational Research.2007 182 (pp127-144). 
Table 1. Reserve Bank of Australia data diagram

\begin{tabular}{|llllllllllllllllllllll}
\hline 4.99 & 5.00 & 5.03 & 5.03 & 5.25 & 5.26 & 5.30 & 5.45 & 5.49 & 5.52 & 5.70 & 5.68 & 5.65 & 5.80 & 6.50 & 6.45 & 6.48 & 6.45 & 6.35 & 6.40 & 6.43 \\
6.43 & 6.44 & 6.45 & 6.48 & 6.40 & 6.35 & 6.40 & 6.30 & 6.32 & 6.35 & 6.13 & 5.70 & 5.58 & 5.18 & 5.18 & 5.17 & 5.15 & 5.21 & 5.23 & 5.05 & 4.65 \\
4.65 & 4.60 & 4.67 & 4.69 & 4.68 & 4.62 & 4.63 & 4.90 & 5.44 & 5.56 & 6.04 & 6.06 & 6.06 & 8.07 & 8.07 & 8.10 & 8.05 & 8.06 & 8.07 & 8.06 \\
8.118 .60 & 10.80 & 11.00 & 11.00 & 11.00 & 9.48 & 9.18 & 8.62 & 8.3 & 8.47 & 8.44 & 8.44 & 8.46 & 8.49 & 8.54 & 8.54 & 8.50 & 8.44 & 8.49 \\
8.40 & 8.46 & 8.50 & 8.50 & 8.47 & 8.47 & 8.47 & 8.48 & 8.48 & 8.54 & 8.56 & 8.39 & 8.89 & 9.91 & 9.89 & 9.91 & 9.91 & 9.90 & 9.88 & 9.86 & 9.86 \\
9.74 & 9.42 & 9.27 & 9.26 & 8.99 & 8.83 & 8.83 & 8.83 & 8.82 & 8.83 & 8.83 & 8.79 & 8.79 & 8.69 & 8.66 & 8.67 & 8.72 & 8.77 & 9.00 & 9.61 & 9.70 \\
9.94 & 9.94 & 9.94 & 9.95 & 9.94 & 9.96 & 9.97 & 10.83 & 10.75 & 11.20 & 11.40 & 11.54 & 11.50 & 11.34 & 11.50 & 11.50 & 11.58 & 12.42 \\
12.8513 .10 & 13.12 & 13.10 & 13.15 & 13.10 & 13.20 & 14.20 & 14.75 & 14.60 & 14.60 & 14.45 & 14.50 & 14.80 & 15.85 & 16.20 \\
16.50 & 16.40 & 16.40 & 16.35 & 16.10 & 13.70 & 13.50 & 14.00 & 12.30 & 12.00 & 14.35 & 14.60 & 12.50 & 12.75 & 13.70 & 13.45 \\
13.55 & 12.60 & 12.00 & 11.00 & 11.60 & 12.05 & 12.35 & 12.70 & 12.45 & 12.55 & 12.20 & 12.10 & 11.15 & 11.85 & 12.10 & 12.50 \\
12.90 & 12.50 & 13.20 & 13.65 & 13.65 & 13.50 & 13.45 & 13.35 & 14.45 & 14.30 & 15.05 & 15.55 & 15.65 & 14.65 & 14.15 & 13.30 \\
12.65 & 12.70 & 12.80 & 14.50 & 15.10 & 15.15 & 14.30 & 14.25 & 14.05 & 14.70 & 15.05 & 14.05 & 13.80 & 13.25 & 13.00 & 12.85 \\
12.60 & 11.80 & 13.00 & 12.35 & 11.45 & 11.35 & 11.55 & 10.85 & 10.90 & 12.30 & 11.70 & 12.05 & 12.30 & 12.90 & 13.05 & 13.30 \\
13.85 & 14.65 & 15.05 & 15.15 & 14.85 & 15.70 & 15.40 & 15.10 & 14.80 & 15.80 & 15.80 & 15.00 & 14.40 & 13.80 & 14.30 & 14.15 \\
14.45 & 14.10 & 14.05 & 13.75 & 13.30 & 13.00 & 12.55 & 12.25 & 11.85 & 11.50 & 11.10 & 11.15 & 10.70 & 10.25 & 10.55 & 10.25 \\
10.30 & 9.60 & 8.40 & 8.20 & 7.25 & 8.35 & 8.25 & 8.30 & 7.40 & 7.15 & 6.35 & 5.65 & 7.40 & 7.20 & 7.05 & 7.10 & 6.85 & 6.50 & 6.25 & 5.95 \\
5.65 & 5.85 & 5.45 & 5.30 & 5.20 & 5.55 & 5.15 & 5.40 & 5.35 & 5.10 & 5.80 & 6.35 & 6.50 & 6.95 & 8.05 & 7.85 & 7.75 & 8.60 &
\end{tabular}

Table 2. DW Test Results

\begin{tabular}{|c|c|c|c|c|c|}
\hline \multicolumn{6}{|c|}{ Ordinary Least Squares Estimates } \\
\hline SSE & & 2774.27594 & DFE & & 306 \\
\hline MSE & & 9.06626 & Root MSE & & 3.01102 \\
\hline $\mathrm{SBC}$ & & 1562.52428 & AIC & & 1555.06408 \\
\hline Regress R-Square & & 0.1933 & Total R-Square & & 0.1933 \\
\hline Durbin-Watson & & 0.0303 & $\operatorname{Pr}<\mathrm{DW}$ & & $<.0001$ \\
\hline \multirow[t]{2}{*}{$\operatorname{Pr}>\mathrm{DW}$} & & 1.0000 & & & \\
\hline & & & Standard & & Approx \\
\hline Variable & DF & Estimate & Error & t Value & $\operatorname{Pr}>|t|$ \\
\hline Intercept & 1 & 7.5194 & 0.3440 & 21.86 & $<.0001$ \\
\hline $\mathrm{t}$ & 1 & 0.0165 & 0.001930 & 8.56 & $<.0001$ \\
\hline
\end{tabular}


Table 3. Estimation of Autocorrelation

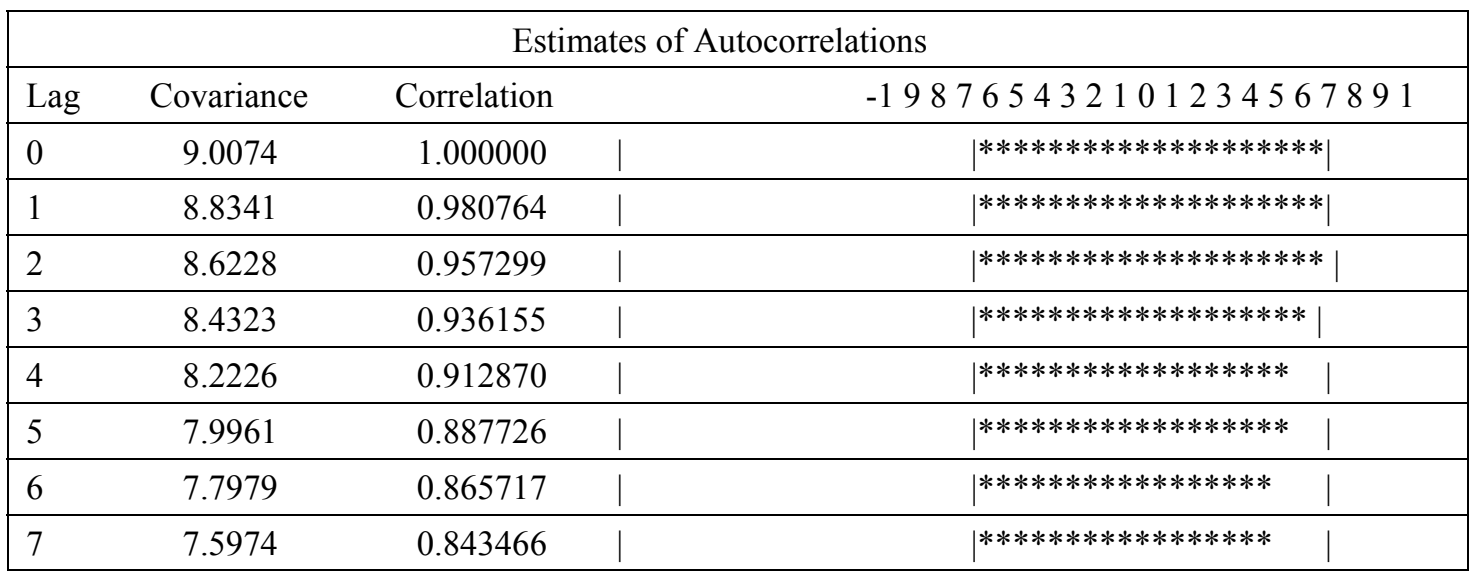

Table 4. PQ and LM Test Results

\begin{tabular}{|cccccc|}
\hline \multicolumn{6}{c|}{ Q and LM Tests for ARCH Disturbances } \\
\hline Order & \multicolumn{2}{c|}{ Q } & Pr $>$ Q & LM & Pr $>$ LM \\
\hline 1 & 286.1017 & $<.0001$ & 283.4412 & $<.0001$ \\
\hline 2 & 545.0776 & $<.0001$ & 283.6751 & $<.0001$ \\
\hline 3 & 782.4323 & $<.0001$ & 283.8243 & $<.0001$ \\
\hline 4 & 995.5900 & $<.0001$ & 284.2103 & $<.0001$ \\
\hline 5 & 1183.6297 & $<.0001$ & 284.2391 & $<.0001$ \\
\hline 6 & 1353.8193 & $<.0001$ & 284.6598 & $<.0001$ \\
\hline 7 & 1507.0917 & $<.0001$ & 284.7027 & $<.0001$ \\
\hline 8 & 1638.3835 & $<.0001$ & 285.3592 & $<.0001$ \\
\hline 9 & 1750.6976 & $<.0001$ & 285.3872 & $<.0001$ \\
\hline 10 & 1842.9918 & $<.0001$ & 286.2225 & $<.0001$ \\
\hline 11 & 1919.1500 & $<.0001$ & 286.5690 & $<.0001$ \\
\hline 12 & 1981.4862 & $<.0001$ & 286.6223 & $<.0001$ \\
\hline
\end{tabular}

Table 5. The fitted-model Results

\begin{tabular}{|c|c|c|c|c|c|}
\hline \multicolumn{6}{|c|}{ The AUTOREG Procedure } \\
\hline \multicolumn{6}{|c|}{ GARCH Estimates } \\
\hline SSE & & 107.927726 & Observations & & 308 \\
\hline MSE & & 0.35041 & Uncond Var & & 041436 \\
\hline Log Likelihood & & -275.54282 & Total R-Squa & & 0.9969 \\
\hline SBC & & 579.736136 & AIC & & 85637 \\
\hline \multirow[t]{2}{*}{ Normality Test } & & 4322.0655 & $\operatorname{Pr}>$ ChiSq & & $<.0001$ \\
\hline & & & Standard & & Approx \\
\hline Variable & $\mathrm{DF}$ & Estimate & Error & t Value & $\operatorname{Pr}>|t|$ \\
\hline $\mathrm{t}$ & 1 & 0.0358 & 0.0285 & 1.26 & 0.2086 \\
\hline AR1 & 1 & -1.0754 & 0.0760 & -14.15 & $<.0001$ \\
\hline AR2 & 1 & 0.0810 & 0.0777 & 1.04 & 0.2971 \\
\hline ARCH0 & 1 & 0.3400 & 0.008580 & 39.63 & $<.0001$ \\
\hline ARCH1 & 1 & $2.554 \mathrm{E}-23$ & $2.352 \mathrm{E}-15$ & 0.00 & 1.0000 \\
\hline GARCH1 & 1 & 0.0298 & 0.003006 & 9.90 & $<.0001$ \\
\hline
\end{tabular}




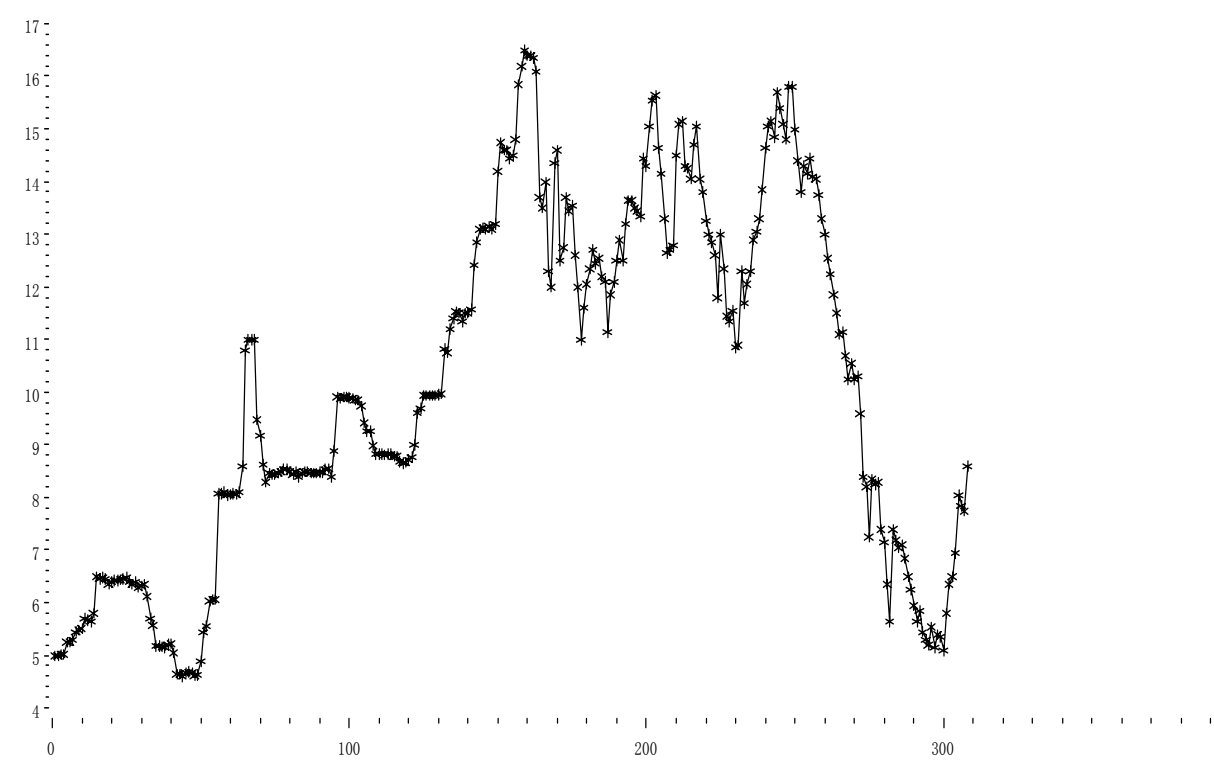

Figure 1. The figure of time series $\left\{x_{t}\right\}$

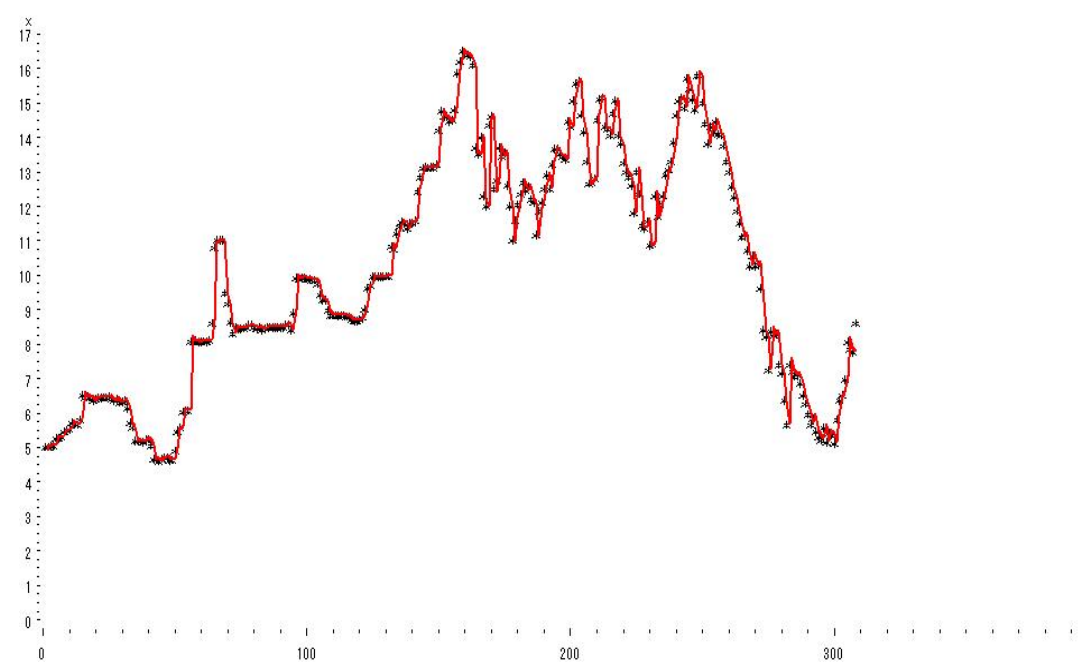

Figure 2. The figure of fitted-model 